\title{
Brain atrophy in paediatric sickle cell anaemia: findings from the silent infarct transfusion (SIT) trial
}

\author{
Jamie M. Kawadler, Chris A. Clark, Robert C. McKinstry, Fenella J. \\ Kirkham
}

Developmental Neurosciences, UCL Institute of Child Health, London, UK

Up to $35 \%$ of children with sickle cell anaemia (SCA) experience silent cerebral infarction (SCI; DeBaun et al, 2012), in which there is a hyperintensity on T2-weighted magnetic resonance imaging (MRI) without focal neurological deficit. Several processes cause SCI, including occlusion by irreversibly sickled cells of small vessels in the arterial borderzones, where flow is relatively low compared with metabolic demand. Compared with controls, MRI shows cortical thinning (Kirk et al, 2009), significant decreases in white matter density extending along the ventricles in an arterial borderzone distribution (Baldeweg et al, 2006) and decrease of volumes of specific subcortical structures (Kawadler et al, 2013), but little is known about long-term brain morphometric changes.

The Silent Infarct Transfusion (SIT) trial (DeBaun et al, 2014) showed, in 196 children with SCA and SCI aged 5-15 years, that blood transfusion reduced recurrence of infarction compared with standard care. This provides the unique opportunity to study intra-subject changes in brain volume over approximately 3 years during childhood and adolescence, with structural image evaluation of T1-weighted anatomical MRI data using normalization of atrophy (SIENA) (Smith et al, 2002), a semi-automated quantification of two time-point whole-brain per cent brain volume change (\%BVC). SIENA allows reliable estimation of longitudinal changes in global brain volume (Smith et al, 2002). To date, there are no studies investigating within-subject longitudinal brain volume change in children with SCA. The primary hypothesis of this exploratory study is that transfusion therapy will have a protective effect on any loss of brain volume and concomitant cognitive function.

Inclusion criteria included: randomization to the SIT trial (i.e. patients with evidence of SCI at baseline), and MRI investigation at pre-randomization and trial exit on a $1.5 \mathrm{~T}$ scanner with a 3D T1-weighted sequence for optimal estimation of atrophy (7 of 29 sites). Analysis included a binary factor to explain variability in whether the same or a different scanner system was used, coded as the same even if the scanner underwent a software upgrade in the interim. SIENA (Smith et al, 2002) estimates \%BVC between two time points in four steps: whole head data are used to extract brain and skull images and are manually checked, the two brain-masked images are aligned to each other and sampled to a halfway space, tissue type segmentation is carried out to find brain/non-brain edge points, and perpendicular edge displacement between the two time-points is estimated. The mean edge displacement is calculated as the global estimate of \%BVC. A negative \%BVC indicates 'atrophy', while a positive \%BVC indicates 'growth' (Fig 1 ). SIENA \%BVC was calculated for each patient for the time lapse between the pre-randomization and exit MRI scans, then calculated as a $\% \mathrm{BVC} /$ year index to control for varying time lapses between scans. A measure of Full-Scale intelligence quotient (FSIQ) was collected from the 4-subtest Wechsler Abbreviated Scale of 
Intelligence at baseline and study exit. Change in FSIQ per year was calculated by the difference in FSIQ scores divided by the time lapse between testing. Statistical analyses were computed with R (http://www.r-project.org/). A model was fitted to the data to examine the influence of treatment group, while controlling for any effect of age, gender, scanner variability, and also the interactions between treatment group, age, gender and scanner variability. Pearson's product moment correlations $(r)$ were carried out to correlations between \%BVC/year and age at pre-randomization and FSIQ.

Demographics and results for the SIENA cohort are displayed in Table 1 . Thirty-two patients with SCD (30 HbSS, $2 \mathrm{HbSB}^{0}$-thalassaemia) were included: 16 on standard treatment, 16 on transfusion. There was a significant difference in gender distribution between the two groups, but no significant difference in age. There were no significant differences in baseline or exit FSIQ. There was no difference in \%BVC/year between the two groups, but there was a significant correlation between age at pre-randomization to the trial and $\% \mathrm{BVC} /$ year, driven by the transfusion group (Table $\underline{1}$, Fig $\underline{1}$ ). Baseline and exit FSIQ assessments were available for 29 patients ( 15 on standard treatment, 14 on transfusion). There were no significant differences in mean FSIQ/year change and residual data of SIENA \%BVC/year (corrected for age, gender and variability in scanner) did not correlate significantly with FSIQ/year change $(r=0.068, P=0.725)$.

Table 1. Demographics and results of SIENA analysis

\section{Standard Treatment $(n=16)$ \\ Transfusion \\ $(n=16)$}

1. MRI, magnetic resonance imaging; FSIQ, Full-Scale intelligence quotient; SD, standard deviation.

2. ${ }^{a}$ available for 29 of 32 subjects who had baseline and exit FSIQ available.

3. ${ }^{b}$ calculated per year (i.e. volume change/time lapse).

\begin{tabular}{|c|c|c|c|}
\hline Gender & $6 \mathrm{M}, 10 \mathrm{~F}$ & $12 \mathrm{M}, 4 \mathrm{~F}$ & $\chi^{2}=4.571, P=0.037$ \\
\hline $\begin{array}{l}\text { Age at Pre-randomization } \\
\text { MRI (years; Mean } \pm \text { SD) }\end{array}$ & $9.41 \pm 2.54$ & $11.06 \pm 2.12$ & $t=-1.99, P=0.055$ \\
\hline \multicolumn{4}{|l|}{ Cognitive Dataa } \\
\hline Baseline FSIQ (Mean \pm SD) & $95.87 \pm 7.78$ & $88.71 \pm 13.56$ & $t=1.725, P=0.096$ \\
\hline Exit FSIQ (Mean \pm SD) & $94.33 \pm 10.30$ & $88.07 \pm 13.34$ & $t=1.428, P=0.164$ \\
\hline FSIQ/year (Mean \pm SD) & $-0.61 \pm 2.38$ & $-0.12 \pm 3.55$ & $t=0.179, P=0.666$ \\
\hline \multicolumn{4}{|l|}{ MRI Analysis } \\
\hline $\begin{array}{l}\text { Time lapse (years) } \\
\text { Mean + SD (range) }\end{array}$ & $\begin{array}{l}3.20 \pm 0.22(2.84- \\
3.84)\end{array}$ & $\begin{array}{l}3.11 \pm 0.13(2.93- \\
3.47)\end{array}$ & \\
\hline $\begin{array}{l}\text { SIENA \% Brain Volume } \\
\text { Changeb Mean } \pm \text { SD } \\
\text { (range) }\end{array}$ & $\begin{array}{l}-0.62 \pm 0.44 \% \\
(-1.80-0.24)\end{array}$ & $\begin{array}{l}-0.96 \pm 1.00 \% \\
(-2.91-0.319)\end{array}$ & $\begin{array}{l}\text { anova } F=0.0012 \\
P=0.972\end{array}$ \\
\hline
\end{tabular}

This study aimed to characterize rates of annual brain growth or atrophy in a subset of children with SCD with existing SCI randomized in the SIT trial. Over approximately a 3year period, there were no significant differences in brain volumetric change between the standard treatment and transfusion arms of the trial, although both showed rates of atrophy beyond previously reported data in healthy children aged 4-18 years (Brain Development Group, 2012). Further, the results show a significant negative correlation between age at 
randomization and $\% \mathrm{BVC} /$ year. The rates of atrophy from this study are greater than the reported $0.15 \%$ error margin (Smith et al, 2002), and those found in healthy adult populations $(-0 \cdot 23 \% \pm 0 \cdot 36 \%)$ (Takao et al, 2012) whilst comparable to previous reports in adults with small vessel disease $(-0 \cdot 914 \% \pm 0 \cdot 16 \%)$ (Nitkunan et al, 2011). Although the SIT trial showed reduction of recurrence of cerebral infarct with transfusion (DeBaun et al, 2014), there is no evidence from this study for a protective effect on progressive cerebral atrophy, perhaps because the mechanisms causing recurrent infarction and progressive atrophy are different.

There are limitations to this study. This subset, of around $16 \%$ of the children enrolled in SIT, had T1-weighted MRI with $1 \mathrm{~mm}$ isotropic resolution, but they are likely to be representative as there are equal numbers in each arm in this randomized trial. Clinical scanner systems may not have stayed the same for the duration of the study. Change in intensity of tissues from different scanner systems may introduce systematic error; however SIENA's mean edge displacement algorithm is relatively insensitive to changes in intensity of tissues between scans (Takao et al, 2011).

In summary, cerebral atrophy over time in children with SCA and SCI occurs at a rate comparable to that seen in small vessel disease in adults, which is apparently greater than that documented in healthy children and adults and is not prevented by transfusion.

\section{Author contributions}

JMK analysed the data and wrote the paper, CAC and RCM contributed to editing the paper, FJK designed the research study and contributed to editing the paper. The authors would like to acknowledge Michael DeBaun, Principal Investigator of the SIT trial, for making the data available.

\section{References}

Baldeweg, T., Hogan, A.M., Saunders, D.E., Telfer, P., Gadian, D.G., Vargha-Khadem, F. \& Kirkham, F.J. (2006) Detecting white matter injury in sickle cell disease using voxel-based morphometry. Annals of Neurology, 59, 662-672.

Brain Development Group (2012) Total and regional brain volumes in a population-based normative sample from 4 to 18 years: the NIH MRI Study of Normal Brain Development. Cerebral Cortex, 22, 1-12.

DeBaun, M.R., Armstrong, F.D., McKinstry, R.C., Ware, R.E., Vichinsky, E. \& Kirkham, F.J. (2012) Silent cerebral infarcts: a review on a prevalent and progressive cause of neurologic injury in sickle cell anemia. Blood, 119, 4587-4596.

DeBaun, M.R., Gordon, M., McKinstry, R.C., Noetzel, M.J., White, D.A., Sarnaik, S.A., Meier, E.R., Howard, T.H., Majumdar, S., Inusa, B.P.D., Telfer, P.T., Kirby-Allen, M., McCavit, T.L., Kamdem, A., Airewele, G., Woods, G.M., Berman, B., Panepinto, J.A., Fuh, B.R., Kwiatkowski, J.L., King, A.A., Fixler, J.M., Rhodes, M.M., Thompson, A.A., Heiny, M.E., Redding-Lallinger, R.C., Kirkham, F.J., Dixon, N., Gonzalez, C.E., Kalinyak, K.A., Quinn, C.T., Strouse, J.J., Miller, J.P., Lehmann, H., Kraut, M.A. Jr, Ball, W.S., Hirtz, D. \& 
Casella, J.F. (2014) Controlled trial of transfusions for silent cerebral infarcts in sickle cell anemia. The New England Journal of Medicine, 371, 699-710.

Kawadler, J.M., Clayden, J.D., Kirkham, F.J., Cox, T.C., Saunders, D.E. \& Clark, C.A. (2013) Subcortical and cerebellar volumetric deficits in paediatric sickle cell anaemia. British Journal of Haematology, 163, 373-376.

Kirk, G.R., Haynes, M.R., Palasis, S., Brown, C., Burns, T.G., McCormick, M. \& Jones, R.A. (2009) Regionally specific cortical thinning in children with sickle cell disease. Cerebral Cortex, 19, 1549-1556.

Nitkunan, A., Lanfranconi, S., Charlton, R.A., Barrick, T.R. \& Markus, H.S. (2011) Brain Atrophy and Cerebral Small Vessel Disease A Prospective Follow-Up Study. Stroke, 42, 133-138.

Smith, S.M., Zhang, Y., Jenkinson, M., Chen, J., Matthews, P.M., Federico, A. \& De Stefano, N. (2002) Accurate, robust, and automated longitudinal and cross-sectional brain change analysis. NeuroImage, 17, 479-489.

Takao, H., Hayashi, N. \& Ohtomo, K. (2011) Effect of Scanner in Longitudinal Studies of Brain Volume Changes. Journal of Magnetic Resonance Imaging, 34, 438-444.

Takao, H., Hayashi, N. \& Ohtomo, K. (2012) A longitudinal study of brain volume changes in normal aging. European Journal of Radiology, 81, 2801-2804. 